\title{
Telomere length is highly repeatable and declines with more elaborate sexual ornamentation in a short-lived passerine
}

\author{
Tereza Kauzálová ${ }^{1}$, Oldřich Tomášek ${ }^{1}$, Ellis Mulder ${ }^{2}$, Simon Verhulst $^{2}$, and Tomas \\ Albrecht $^{1}$ \\ ${ }^{1}$ Institute of Vertebrate Biology Czech Academy of Sciences \\ ${ }^{2}$ University of Groningen
}

May 22, 2021

\begin{abstract}
Quantifying an individual's state as fitness proxy has proven challenging, but accumulating evidence suggests that telomere length and attrition may indicate individual somatic state and success at self-maintenance, respectively. Sexual ornamentation is also thought to signal phenotypic quality, but links between telomeres and sexual ornamentation have been little explored. To address this issue, we examined whether telomere length and dynamics are predicted by the expression of a sexually selected ornament, the length of outermost tail feathers (streamers), using longitudinal data from a population of European barn swallows (Hirundo rustica). We further assessed associations of telomere length with age, sex, breeding status and survival. Telomere length showed high individual repeatability $(\mathrm{R}=0.97)$ across years while shortening with age in both sexes. Telomere length and dynamics were not significantly associated with survival to the next year, remaining lifespan or reproduction status (comparing breeding and non-breeding yearlings). Tail streamer length was negatively associated with telomere length, independent of sex. Thus, telomere length may reflect the costs of carrying an elaborated sexual ornament, although ornament size did not significantly predict telomere shortening. In conclusion, telomere length in adult barn swallows is a highly consistent trait and shows a negative relationship with sexual ornamentation, suggesting a trade-off between sexual ornamentation and telomere length.
\end{abstract}

\section{Hosted file}

Kauzalova-etal_MolEcol.doc available at https://authorea.com/users/415127/articles/ 523039-telomere-length-is-highly-repeatable-and-declines-with-more-elaborate-sexualornamentation-in-a-short-lived-passerine 